\title{
PESQUISA DE Listeria spp. E Salmonella spp. ISOLADAS DE TILÁPIAS (Oreochromis spp.) OBTIDAS DE PESQUE-PAGUES NO INTERIOR DO ESTADO DE SÃO PAULO, BRASIL
}

\author{
RESEARCH OF Listeria spp. AND Salmonella spp. ISOLATED FROM TILAPIAS \\ (Oreochromis spp.) OBTAINED FROM FEE FISHING IN SÃO PAULO STATE, BRAZIL
}

\author{
T. D. $\operatorname{COSTA}^{1 *}$, A. M. C. VIDAL ${ }^{1}$, A. C. N. VAZ ${ }^{2}$
}

\begin{abstract}
RESUMO
$\mathrm{O}$ aparecimento das infecções e intoxicações alimentares associadas aos peixes está ligado às condições higiênicas, sanitárias e ao baixo índice de conhecimento das boas práticas de manipulação, por isso pesquisas relacionadas ao assunto são essenciais na literatura. Este estudo objetivou verificar a presença de Listeria spp. e Salmonella spp. em filés de tilápias (Oreochromis spp.) e de peças inteiras do mesmo peixe em pesquepagues da microrregião interiorana do Estado de São Paulo, bem como realizar o antibiograma de tais isolados. Para tal, foram obtidos 36 exemplares de tilápia, sendo que após o abate e filetagem no próprio estabelecimento, os peixes $(\mathrm{n}=18)$ e os filés $(\mathrm{n}=18)$ foram submetidos ao isolamento de Listeria spp e Salmonella spp. Foram encontrados dos materiais analisados, 13 (36,12\%) isolados de Listeria spp., sendo 7 $(53,85 \%)$ isolados do peixe ínteiro e $6(46,15 \%)$ isolados dos filés, além do resultados dos testes de sensibilidade antimicrobiana in vitro, no qual mostra que os medicamentos mais recomendados foram: gentamicina > ampicilina/neomicina > florfenicol > tetraciclina > cefoperazona > ciprofloxacina > cefalotina. Já para Salmonella spp. foi verificado $21(58,34 \%)$ isolados, sendo $11(61,12 \%)$ presentes nas amostras de filés e $10(55,56 \%)$ nas tilápias inteiras. Sobre o comprotamento dos antimicrobianos frente às Salmonella spp. constatou-se maiores taxas de sensibilidade a gentamicina $(95,24 \%)$, amicacina $(66,67 \%)$ e ciprofloxacina $(66,67 \%)$. Esses resultados servem de alerta, pois a veiculação desses micro-organismos potencialmente patogênicos em tilápias, peixe de grande valor alimentício e zootécnico nos pesque-pagues, pode vir a ser a causa de grandes problemas na saúde pública, além disso, o uso indiscriminado de antibióticos veterinários tem contribuído para a resistência entre os isolados, havendo necessidade de critérios no uso de antimicrobianos nos tanques de criação.
\end{abstract}

PALAVRAS-CHAVE: ANTIBIOGRAMA. INSPEÇÃO. Listeria spp.. TILÁPIA. Salmonella spp.

AGRADECIMENTOS: À Coordenação de Aperfeiçoamento de Pessoal de Nível Superior (CAPES), pelo apoio financeiro e a Faculdade de Zootecnia e Engenharia de Alimentos da Universidade de São Paulo (FZEA/USP), por ter cedido o espaço para realização dos testes microbiológicos.

ÁREA TEMÁTICA: Higiene e Inspeção de Produtos de Origem Animal

\footnotetext{
${ }^{1}$ Faculdade de Ciências Agrárias e Veterinárias da Universidade Estadual Paulista (UNESP) - Câmpus de Jaboticabal

${ }^{2}$ Faculdade de Ciências Agrárias e Veterinárias da Universidade Estadual Paulista (UNESP) - Câmpus de Jaboticabal

* thatayadc@gmail.com
} 